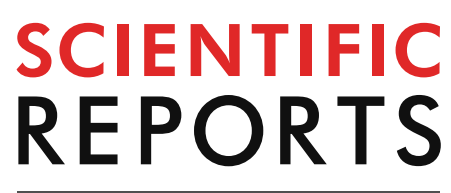

natureresearch

Check for updates

\title{
A generalized heat conduction model of higher-order time derivatives and three-phase-lags for non-simple thermoelastic materials
}

\begin{abstract}
Ahmed E. Abouelregal ${ }^{1,2 \bowtie}$, K. M. Khalil ${ }^{1,3}$, F. A. Mohammed ${ }^{1,4}$, M. E. Nasr ${ }^{1,3}$, Adam Zakaria $^{1,5}$ \& Ibrahim-Elkhalil Ahmed ${ }^{1,6}$

In the current work, a new generalized model of heat conduction has been constructed taking into account the influence of the microscopic structure into the on non-simple thermoelastic materials. The new model was established on the basis of the system of equations that includes three-phase lags of higher-order and two different temperatures, namely thermodynamic and conductive temperature. The two-temperature thermoelastic model presented by Chen and Gurtin ( $Z$ Angew Math Phys 19(4):614-627, 1968) and some other previous models have been introduced as special cases from the proposed model. As an application of the new model, we studied the thermoelastic interactions resulting from sudden heating in an isotropic solid subjected to external body force. The influence of the discrepancy parameter and higher-order of the time-derivative has been discussed. This work will enable future investigators to gain insight into non-simple thermoelasticity with different phase delays of higher-order in detail.
\end{abstract}

$\begin{array}{ll}\text { List of symbols } & \\ \lambda, \mu & \text { Lamés constants } \\ \alpha_{t} & \text { Thermal expansion coefficient } \\ C_{e} & \text { Specific heat } \\ \gamma=(3 \lambda+2 \mu) \alpha_{t} & \text { Thermal coupling parameter } \\ T_{0} & \text { Environmental temperature } \\ \theta=T-T_{0} & \text { Temperature increment } \\ T & \text { Absolute temperature } \\ \boldsymbol{u} & \text { Displacement vector } \\ e=\text { divu } & \text { Cubical dilatation } \\ \sigma_{i j} & \text { Stress tensor } \\ e_{i j} & \text { Strain tensor } \\ \boldsymbol{q} & \text { Heat flux vector } \\ K & \text { Thermal conductivity } \\ \rho & \text { Material density } \\ Q & \text { Heat source } \\ t & \text { The time } \\ \delta_{i j} & \text { Kronecker's delta function }\end{array}$

${ }^{1}$ Department of Mathematics, College of Science and Arts, Jouf University, Al-Qurayyat, Saudi Arabia. ${ }^{2}$ Department of Mathematics, Faculty of Science, Mansoura University, Mansoura 35516, Egypt. ${ }^{3}$ Mathematics Department, Faculty of Science, Benha University, P.O. 13518, Benha, Egypt. ${ }^{4}$ Department of Mathematics, South Valley University, Qena, Egypt. ${ }^{5}$ Department of Mathematics, Faculty of Science, University of Kordofan, El-Obeid, Sudan. ${ }^{6}$ Departement of Mathematics, Faculty of Science and Technologe, Shendi University, Shendi, Sudan. ${ }^{\varpi}$ email: ahabogal@gmail.com 


$\begin{array}{ll}\varphi & \text { Conductive temperature } \\ \tau_{q} & \text { Phase lag of heat flux } \\ \tau_{\theta} & \text { Phase lag of temperature } \\ \tau_{\varphi} & \text { Phase lag of } \nabla \varphi \\ \tau_{0} & \text { Thermal relaxation time } \\ m, n, p & \text { Higher orders } \\ S & \text { The entropy }\end{array}$

Duhamel (1837) was the first to suggest entering the coupling term in the heat equation and proposing the coupling between the temperature and the deformation. However, the heat equation was not well established by the thermodynamic process. Biot ${ }^{1}$ attempted the thermodynamic justification for this equation. The heat equation includes, in this case, the dilatation term depend on the thermodynamics of the non-inverse process. The classical heat conduction theory in thermoelastic solids is based on the assumption that the heat flow is proportional to the temperature gradient. Based on this assumption, the heat equation is governed by a parabolic system of partial differential equations, which predicts that the thermal disturbance in the material will immediately affect all points of the body. The phenomenon of infinite velocity of heat waves conflicts with physical phenomena.

To overcome this defect, generalized thermodynamic theories were introduced. Lord and Shulman ${ }^{2}$ introduced a generalized theory of thermoelasticity that provides one relaxation time and thus the system that governs the heat equation has turned into a hyperbolic type. Green and Lindsay ${ }^{3}$ proposed another model involved two relaxation times.

The following generalization of the theory of thermoelasticity is known as the dual-phase-delay model which was improved by $\mathrm{Tzou}^{3}$ and Chandrasekharaiah ${ }^{4}$. $\mathrm{Tzou}^{3}$ reflected a constitutive equation to explain the lagging behavior in the heat conduction in solid materials. Tzou ${ }^{5,6}$ presented a thermoelastic model including dualphase-delay to the heat flux vector and the temperature gradient.

The two-temperature theory (2TTE), proposed by Chen and Gurtin ${ }^{7}$, Gurtin and Williams ${ }^{8,9}$ and Chen et al. ${ }^{10,11}$, based on two distinct temperatures; conductive $\varphi$ and the thermodynamic $\theta$ temperatures. According to this theory, the difference between these two temperatures is proportional to the applied heat source. Also, in the absence of the heat source, the two temperatures are equal ${ }^{12}$. Based on this theory of thermoelasticity with two-temperature, several authors have studied several problems of thermoelasticity ${ }^{13-18}$. Quintanilla ${ }^{19}$ discussed the structural stability, existence, and spatial behavior of the solution of some problems in 2TT. For isotropic and homogeneous bodies, Yousse ${ }^{20}$ introduced the theory of thermoelasticity with relaxation time and twotemperature. Mukhopadhyay et al. ${ }^{21}$ also, extended generalized thermoelasticity with two temperatures and dual-phase-lag. The two temperatures theory has attracted a lot of attention in the recent years ${ }^{22-26}$.

Recently, many efforts have been introduced to modify the classical heat conduction law. In one of these efforts, Abouelregal ${ }^{27-29}$ introduced some generalized models of heat conduction including higher-order timederivative. Earlier, Chiriţă $\breve{3}^{30}$ investigated the high-order Fourier law to illustrate the lagging performance of heat transfer.

The current paper is concerned with a generalized model that combines the two-temperature theory and the heat conduction of higher-order time-derivatives with two-phase-lags. To further study the accuracy of the current model, the model was applied to study the interaction in an isotropic solid exposed to external body force and due to sudden heating. Some special cases of concern were also deduced from the proposed model. For further clarification and comparison, the numerical results were tabulated and illustrated graphically. The effects of the parameters of temperature distinction, phase delay, and high-orders on all field variables inside the material have been studied. The numerical results obtained in this paper were found to be as good as with the results in the current literature. Also, the results and observations also showed that the analytical solutions correspond well with numerical solutions.

\section{Derivation of the modified model}

The Fourier's law ${ }^{31}$, is the closest model representing heat conduction, which assumes that there is a linear relationship between the heat flow $\boldsymbol{q}$ and the temperature gradient $\nabla \theta$ on the following form:

$$
\boldsymbol{q}(\boldsymbol{x}, t)=-K \nabla \theta(\boldsymbol{x}, t)
$$

The following relations are achieved by the increment in entropy $S$

$$
\begin{gathered}
\operatorname{div} \boldsymbol{q}+Q=-\rho T_{0} \dot{\boldsymbol{S}} \\
\rho T_{0} S=\rho C_{e} \theta+\gamma T_{0} \operatorname{div} \boldsymbol{u}
\end{gathered}
$$

From Eqs. (2) and (3), we obtain the energy equation as

$$
\rho C_{e} \frac{\partial \theta}{\partial t}+\gamma T_{0} \frac{\partial}{\partial t}(\operatorname{div} \boldsymbol{u})=-\operatorname{div} \boldsymbol{q}+Q
$$

Among the governing equations in the two-temperature model (2TT), in addition to the energy Eq. (4), the equation that connecting the two temperatures $\theta$ and $\varphi$ is given by ${ }^{7-12}$ :

$$
\theta=\left(1-a \nabla^{2}\right) \varphi
$$

which, $a>0$ is the temperature distinction parameter (two-temperature). 
The classical Fourier's law (1) has been replaced, according to Quintanilla ${ }^{29}$ with

$$
\boldsymbol{q}(\boldsymbol{x}, t)=-K \nabla \varphi(\boldsymbol{x}, t)
$$

In the DPL model, the heat Eq. (6) is modified by introducing two phase-lags as ${ }^{11}$ :

$$
\boldsymbol{q}\left(\boldsymbol{x}, t+\tau_{q}\right)=-K \nabla \varphi\left(\boldsymbol{x}, t+\tau_{\varphi}\right)
$$

In the above equation, $\tau_{q}$ and $\tau_{\varphi}$ are respectively the phase lags of the heat flux and conductive temperature gradient.

Introducing the phase lag of the temperature $\tau_{\theta}$, in addition to the phase lag $\tau_{q}$ of conductive temperature, we get the following relation

$$
\theta\left(\boldsymbol{x}, t+\tau_{\theta}\right)=\left(1-a \nabla^{2}\right) \varphi\left(\boldsymbol{x}, t+\tau_{\varphi}\right)
$$

Taylor-series-expansion is applied to both sides of Eqs. (7) and (8) and maintain the terms up to a suitable higher-order of time-deferential $(m, n, p)$ in $\tau_{q}, \tau_{\varphi}$, and $\tau_{\theta}$ respectively, to acquire

$$
\begin{gathered}
\left(1+\sum_{r=1}^{m} \frac{\tau_{q}^{r}}{r !} \frac{\partial^{r}}{\partial t^{r}}\right) \boldsymbol{q}=-K\left(1+\sum_{r=1}^{n} \frac{\tau_{\varphi}^{r}}{r !} \frac{\partial^{r}}{\partial t^{r}}\right) \nabla \varphi \\
\left(1+\sum_{r=1}^{p} \frac{\tau_{\theta}^{r}}{r !} \frac{\partial^{r}}{\partial t^{r}}\right) \theta=\left(1-a \nabla^{2}\right)\left(1+\sum_{r=1}^{n} \frac{\tau_{\varphi}^{r}}{r !} \frac{\partial^{r}}{\partial t^{r}}\right) \varphi
\end{gathered}
$$

It was highlighted that the proposed models associated with the higher-order time-differential have been extensively considered in many papers regarding their thermodynamic consistency and also with regard to wellpresented issues and stimulating stability ${ }^{32-37}$. If we adjoin Eq. (9) with the energy Eq. (4), we get

$$
K\left(1+\sum_{r=1}^{n} \frac{\tau_{\varphi}^{r}}{r !} \frac{\partial^{r}}{\partial t^{r}}\right) \nabla^{2} \varphi=\left(1+\sum_{r=1}^{m} \frac{\tau_{q}^{r}}{r !} \frac{\partial^{r}}{\partial t^{r}}\right)\left(\rho C_{e} \dot{\theta}+\gamma T_{0} \dot{e}-\rho Q\right)
$$

By studying a system of equations similar to the foundational equations of type (9) or (10), Chiriţă et al. ${ }^{35}$ explain that there are some restrictions to choosing the higher orders $m, n$, and $p$, for example when $m \geq 5$ leads to an unstable system, and therefore cannot describe a real physical state.

In addition, the field equations, the constitutive relations and the train-displacement relation to thermoelastic isotropic materials at uniform environmental temperature $T_{0}$ are:

$$
\begin{gathered}
\sigma_{i j}=2 \mu e_{i j}+\delta_{i j}\left[\lambda e_{i j}-\gamma \theta\right] \\
2 e_{i j}=u_{j, i}+u_{i, j} \\
\mu u_{i, j j}+(\lambda+\mu) u_{j, i j}-\gamma \theta_{, i}+F_{i}=\rho \ddot{u}_{i}
\end{gathered}
$$

\section{Application to the model}

As to achieve the accuracy of the presented model, we are now studying a thermoelastic body that is exposed to thermal shock and is affected by an external force. It is assumed that all field variables depend only on the distance $x$ and instant time $t$. Then displacement components have the form

$$
u_{x}=u(x, t), \quad u_{y}=0, \quad u_{z}=0 .
$$

The non-zero strain is given by

$$
e=\frac{\partial u(x, t)}{\partial x}
$$

The components of the external body strength can be chosen as

$$
F_{x}=e^{-\omega x}, \quad \omega>0, \quad F_{y}=0, \quad F_{z}=0 .
$$

Equations (9), (11), (12) and (14) then reduce to

$$
\begin{gathered}
(\lambda+2 \mu) \frac{\partial^{2} u}{\partial x^{2}}-\gamma \frac{\partial \theta}{\partial x}+\rho e^{-\omega x}=\rho \frac{\partial^{2} u}{\partial t^{2}} \\
K\left(1+\sum_{r=1}^{n} \frac{\tau_{\varphi}^{r}}{r !} \frac{\partial^{r}}{\partial t^{r}}\right) \frac{\partial^{2} \varphi}{\partial x^{2}}=\left(1+\sum_{r=1}^{m} \frac{\tau_{q}^{r}}{r !} \frac{\partial^{r}}{\partial t^{r}}\right)\left(\rho C_{e} \frac{\partial \theta}{\partial t}+\gamma T_{0} \frac{\partial^{2} u}{\partial t \partial x}\right)
\end{gathered}
$$




$$
\begin{aligned}
\left(1+\sum_{r=1}^{p} \frac{\tau_{\theta}^{r}}{r !} \frac{\partial^{r}}{\partial t^{r}}\right) \theta & =\left(1-a \frac{\partial^{2}}{\partial x^{2}}\right)\left(1+\sum_{r=1}^{n} \frac{\tau_{\varphi}^{r}}{r !} \frac{\partial^{r}}{\partial t^{r}}\right) \varphi \\
\sigma_{x x}=\sigma & =(\lambda+2 \mu) \frac{\partial u}{\partial x}-\gamma \theta
\end{aligned}
$$

We will consider the dimensionless quantities:

$$
\begin{aligned}
& \left\{x^{\prime}, u^{\prime}\right\}=\eta c_{0}\{x, u\}, \quad \sigma^{\prime}=\frac{\sigma}{\lambda+2 \mu},\left\{t^{\prime}, \tau_{q}^{\prime}, \tau_{\theta}^{\prime}, \tau_{\varphi}^{\prime}\right\}=c_{0}^{2} \eta\left\{t, \tau_{0}, \tau_{1}, \tau_{\theta}, \tau_{q}\right\}, \\
& a^{\prime}=\eta^{2} c_{0}^{2} a, \theta^{\prime}=\frac{\gamma}{\lambda+2 \mu} \theta, F^{\prime}=\frac{\rho}{\eta c_{0}(\lambda+2 \mu)} F, c_{0}^{2}=\frac{(\lambda+2 \mu)}{\rho}, \eta=\frac{\rho C_{e} c_{0}^{2}}{K} .
\end{aligned}
$$

The governing Eqs. (18)-(21), by using Eq. (22) may be reformulated in the non-dimensional forms as (neglecting the primes):

$$
\begin{gathered}
\frac{\partial^{2} u}{\partial x^{2}}-\frac{\partial \theta}{\partial x}+e^{-\omega x}=\frac{\partial^{2} u}{\partial t^{2}} \\
\left(1+\sum_{r=1}^{n} \frac{\tau_{\varphi}^{r}}{r !} \frac{\partial^{r}}{\partial t^{r}}\right) \frac{\partial^{2} \varphi}{\partial x^{2}}=\left(1+\sum_{r=1}^{m} \frac{\tau_{q}^{r}}{r !} \frac{\partial^{r}}{\partial t^{r}}\right)\left(\frac{\partial \theta}{\partial t}+\varepsilon \frac{\partial^{2} u}{\partial t \partial x}\right), \\
\left(1+\sum_{r=1}^{p} \frac{\tau_{\theta}^{r}}{r !} \frac{\partial^{r}}{\partial t^{r}}\right) \theta=\left(1-a \frac{\partial^{2}}{\partial x^{2}}\right)\left(1+\sum_{r=1}^{n} \frac{\tau_{\varphi}^{r}}{r !} \frac{\partial^{r}}{\partial t^{r}}\right) \varphi, \\
\sigma=\frac{\partial u}{\partial x}-\theta
\end{gathered}
$$

where $\varepsilon=\frac{\gamma^{2} T_{0}}{\rho^{2} c_{0}^{2} C_{e}}$.

Homogeneous initial conditions are assumed to be

$$
\begin{aligned}
& \theta(x, 0)=\frac{\partial^{r} \theta(x, 0)}{\partial t^{r}}=0, \quad \varphi(x, 0)=\frac{\partial^{r} \varphi(x, 0)}{\partial t^{r}}=0, \\
& u(x, 0)=\frac{\partial^{r} u(x, 0)}{\partial t^{r}}=0, \quad r=\{m, n, p\} .
\end{aligned}
$$

From the description of the previous problem, we find that the boundary conditions are in the form

$$
\begin{aligned}
& \sigma(0, t)=0 \\
& \varphi(0, t)=\varphi_{0} H(t)
\end{aligned}
$$

where the parameter $\varphi_{0}$ is constant and $H(t)$ denotes the Heaviside unit step function.

\section{Solution of the problem}

To get the solution of the problem, we perform the Laplace transform described by

$$
\bar{f}(x, t)=\int_{0}^{\infty} f(x, t) e^{-s t} d t
$$

Transforming Eqs. (23)-(26), we obtain

$$
\begin{gathered}
s^{2} \bar{u}=\mathrm{D}^{2} \bar{u}-\mathrm{D} \bar{\theta}+\frac{1}{s} e^{-\omega x} \\
\ell_{\varphi} \mathrm{D}^{2} \bar{\varphi}=\ell_{q}(\bar{\theta}+\varepsilon \mathrm{D} \bar{u}), \\
\ell_{\theta} \bar{\theta}=\ell_{\varphi}\left(1-a \mathrm{D}^{2}\right) \bar{\varphi}, \\
\bar{\sigma}=\mathrm{D} \bar{u}-\bar{\theta}
\end{gathered}
$$

where 


$$
\begin{aligned}
& \ell_{q}=1+\sum_{r=1}^{m} \frac{\tau_{q}^{r}}{r !} s^{r}, \ell_{\theta}=1+\sum_{r=1}^{p} \frac{\tau_{\theta}^{r}}{r !} s^{r} \\
& \ell_{\varphi}=1+\sum_{r=1}^{n} \frac{\tau_{\varphi}^{r}}{r !} s^{r}, \quad D=\frac{\mathrm{d}}{\mathrm{d} x} .
\end{aligned}
$$

Eliminating $\bar{\theta}$ from Eqs. (30)-(32), we obtain

$$
\begin{gathered}
\left(\mathrm{D}^{2}-s^{2}\right) \bar{e}=\alpha_{1}\left(\mathrm{D}^{2}-a \mathrm{D}^{4}\right) \bar{\varphi}+\frac{\omega}{s} e^{-\omega x} \\
{\left[\mathrm{D}^{2}-\alpha_{2}\right] \bar{\varphi}=\alpha_{3} \bar{e}} \\
\bar{\sigma}=\bar{e}-\alpha_{1}\left(1-a \mathrm{D}^{2}\right) \bar{\varphi}
\end{gathered}
$$

where

$$
\alpha_{1}=\frac{\ell_{\varphi}}{\ell_{\theta}}, \quad \alpha_{2}=\frac{\ell_{q}}{a \ell_{q}+\ell_{\theta}}, \quad \alpha_{3}=\frac{\varepsilon \ell_{\theta} \ell_{q}}{a \ell_{q} \ell_{\varphi}+\ell_{\varphi} \ell_{\theta}} .
$$

Eliminating $\bar{e}$ between Eqs. (35) and (36), we get

$$
\left(\mathrm{D}^{4}-A \mathrm{D}^{2}+B\right) \bar{\varphi}=\alpha_{4} e^{-\omega x}
$$

where

$$
\mathrm{A}=\frac{s^{2}+\alpha_{2}+\alpha_{1} \alpha_{3}}{1+a \alpha_{1} \alpha_{3}}, \mathrm{~B}=\frac{s^{2} \alpha_{2}}{1+a \alpha_{1} \alpha_{3}}, \alpha_{4}=\frac{\omega \alpha_{3}}{s\left(1+a \alpha_{1} \alpha_{3}\right)} .
$$

The general solution of Eq. (39) which is bounded as $x \rightarrow \infty$ is given by

$$
\bar{\varphi}(x)=C_{1} \mathrm{e}^{-m_{1} x}+C_{2} \mathrm{e}^{-m_{2} x}+C_{3} e^{-\omega x}
$$

where $C_{3}=\alpha_{4} /\left(\omega^{4}-A \omega^{2}+B\right)$ and $C_{1}$ and $C_{2}$ are some parameters.

Also, the parameters $m_{1}$ and $m_{1}$ are the roots of the equation

$$
m^{4}-A m^{2}+B=0
$$

Substituting (41) into Eqs. (36) and (32), we obtain

$$
\begin{gathered}
\bar{e}(x)=\frac{\left(m_{1}^{2}-\alpha_{2}\right)}{\alpha_{3}} C_{1} \mathrm{e}^{-m_{1} x}+\frac{\left(m_{2}^{2}-\alpha_{2}\right)}{\alpha_{3}} C_{2} \mathrm{e}^{-m_{2} x}+\frac{\left(\omega^{2}-\alpha_{2}\right)}{\alpha_{3}} C_{3} e^{-\omega x} \\
\bar{\theta}(x)=\alpha_{1}\left(1-a m_{1}^{2}\right) C_{1} \mathrm{e}^{-m_{1} x}+\alpha_{1}\left(1-a m_{2}^{2}\right) C_{2} \mathrm{e}^{-m_{2} x} \\
+\alpha_{1}\left(1-a \omega^{2}\right) C_{3} e^{-\omega x}
\end{gathered}
$$

Introducing Eq. (43) into Eq. (16), we get

$$
\bar{u}(x)=-\frac{\left(m_{1}^{2}-\alpha_{2}\right)}{m_{1} \alpha_{3}} C_{1} \mathrm{e}^{-m_{1} x}-\frac{\left(m_{2}^{2}-\alpha_{2}\right)}{m_{2} \alpha_{3}} C_{2} \mathrm{e}^{-m_{2} x}-\frac{\left(\omega^{2}-\alpha_{2}\right)}{\omega \alpha_{3}} C_{3} e^{-\omega x}
$$

Using Eqs. (41) and (43) into Eq. (37) we have

$$
\begin{aligned}
\bar{\sigma}(x)= & {\left[\frac{\left(m_{1}^{2}-\alpha_{2}\right)}{\alpha_{3}}-\alpha_{1}\left(1-a m_{1}^{2}\right)\right] C_{1} \mathrm{e}^{-m_{1} x} } \\
& +\left[\frac{\left(m_{2}^{2}-\alpha_{2}\right)}{\alpha_{3}}-\alpha_{1}\left(1-a m_{2}^{2}\right)\right] C_{2} \mathrm{e}^{-m_{2} x} \\
& +\left[\frac{\left(\omega^{2}-\alpha_{2}\right)}{\alpha_{3}}-\alpha_{1}\left(1-a \omega^{2}\right)\right] C_{3} e^{-\omega x}
\end{aligned}
$$

By taking the Laplace transform to the boundary conditions (28), we get

$$
\begin{array}{lll}
\bar{\sigma}=0 & \text { on } & x=0 \\
\bar{\varphi}=\varphi_{0} / s & \text { on } & x=0
\end{array}
$$

Substituting the functions of $\bar{\sigma}$ and $\bar{\varphi}$ given in (41) and (46) into the boundary conditions (47), we can obtain the integral parameters $C_{1}$ and $C_{2}$. 


\section{Special cases}

- The classical coupled thermoelasticity $(\mathrm{CTE})^{1}$ is yielded when $\tau_{q}=\tau_{\theta}=\tau_{\varphi}=0, a=0, \theta=\varphi$. In this case, the heat conduction equation can be expressed as

$$
K \nabla^{2} \theta=\rho C_{e} \frac{\partial \theta}{\partial t}+\gamma T_{0} \frac{\partial e}{\partial t}-\rho Q
$$

- Lord-Shulman theory (LS) ${ }^{38}$ is given by setting $\tau_{q}=\tau_{0}>0, a=0, \theta=\varphi, \tau_{\theta}, \tau_{\varphi} \rightarrow 0$ and taken $m=1$. In this case, the heat equation has the form

$$
K \nabla^{2} \theta=\left(1+\tau_{0} \frac{\partial}{\partial t}\right)\left(\rho C_{e} \frac{\partial \theta}{\partial t}+\gamma T_{0} \frac{\partial e}{\partial t}-\rho Q\right)
$$

- The heat equation proposed by Tzou (DPL) $)^{3,6}$ is given when $a=0, \theta=\varphi, \tau_{\theta}=\tau_{\varphi}, n=1, m=2$ and take the following the form

$$
K\left(1+\tau_{\theta} \frac{\partial}{\partial t}\right) \nabla^{2} \theta=\left(1+\tau_{q} \frac{\partial}{\partial t}+\frac{\tau_{q}^{2}}{2} \frac{\partial^{2}}{\partial t^{2}}\right)\left(\rho C_{e} \frac{\partial \theta}{\partial t}+\gamma T_{0} \frac{\partial e}{\partial t}-\rho Q\right)
$$

- Tzou $^{3}$ and Chandrasekharaiah ${ }^{4}$ models with dual-phase-lags (DPL) are obtained by setting $a=0, \theta=\varphi$, $\tau_{\theta}=\tau_{\varphi}, m=n=1$. The heat equation in this case is given by

$$
K\left(1+\tau_{\theta} \frac{\partial}{\partial t}\right) \nabla^{2} \theta=\left(1+\tau_{q} \frac{\partial}{\partial t}\right)\left(\rho C_{e} \frac{\partial \theta}{\partial t}+\gamma T_{0} \frac{\partial e}{\partial t}-\rho Q\right)
$$

- The two-temperature thermoelasticity model with phase-lags introduced by Mukhopadhyay et al. ${ }^{21}$ (MTTE) is given when we take $a>0, \tau_{\theta}=\tau_{\varphi}, m=n=p=1$. In this case, the two-temperature relation and the heat equation are:

$$
\begin{gathered}
\theta=\left(1-a \nabla^{2}\right) \varphi \\
K\left(1+\tau_{\theta} \frac{\partial}{\partial t}\right) \nabla^{2} \varphi=\left(1+\tau_{q} \frac{\partial}{\partial t}\right)\left(\rho C_{e} \frac{\partial \theta}{\partial t}+\gamma T_{0} \frac{\partial e}{\partial t}-\rho Q\right)
\end{gathered}
$$

- The governing equations proposed by ${ }^{20}$ (YTTE) can be acquired as special case by taking $a>0, \tau_{\theta}=\tau_{\varphi}=0$, $\tau_{q}=\tau_{0}>0, m=n=p=1$. In this case

$$
\begin{gathered}
\theta=\left(1-a \nabla^{2}\right) \varphi \\
K \nabla^{2} \varphi=\left(1+\tau_{0} \frac{\partial}{\partial t}\right)\left(\rho C_{e} \frac{\partial \theta}{\partial t}+\gamma T_{0} \frac{\partial e}{\partial t}-\rho Q\right)
\end{gathered}
$$

- The generalized two-temperature thermoelasticity theory with two-phase-lags and high-order (HTTE) is obained when $a>0, \tau_{q}, \tau_{\theta}, \tau_{\varphi}>0, n, m, p \geq 1$.

\section{Numerical results}

In the current section, we will try to provide a practical example to validate the accuracy of the current model. Also, the results can be scheduled to support other researchers to compare their results and verify their accuracy. For the purposes of numerical discussions, we have taken the values of the copper material constants as ${ }^{39}$ :

$$
\begin{aligned}
& C_{E}=383.1\left(\frac{\mathrm{J}}{\mathrm{kg} \mathrm{K}}\right), \quad T_{0}=293(\mathrm{~K}), \quad \alpha_{t}=1.78 \times 10^{-5}\left(\frac{1}{\mathrm{~K}}\right), \quad K=386\left(\frac{\mathrm{W}}{\mathrm{m} \mathrm{K}}\right), \\
& \lambda=7.76 \times 10^{10}\left(\frac{\mathrm{N}}{\mathrm{m}^{2}}\right), \quad \mu=3.86 \times 10^{10}\left(\frac{\mathrm{N}}{\mathrm{m}^{2}}\right), \quad \rho=8954\left(\frac{\mathrm{kg}}{\mathrm{m}^{3}}\right) .
\end{aligned}
$$

We performed the calculations when $t=0.12 \mathrm{~s}, \varphi_{0}=1$, and $\varepsilon=0.0168$. By observing previous literature, most researchers have addressed such a problem without providing tabular results. They provide only some graphical examples to explain and clarify the phenomena.

To obtain the solutions for the distributions of conductive and dynamical temperatures, stress, strain displacement fields in the real domain, we have to employ a numerical inversion technique of the Laplace transform to Eqs. (43)-(46) respectively. Details of these techniques can be found in Honig and Hirdes ${ }^{40}$. In this technique, any function $\bar{g}(x, s)$ in Laplace domain can be inverted to the time domain $g(x, t)$ numerically by the relation

$$
g(x, t)=\frac{e^{\omega t}}{t}\left(\frac{1}{2} \bar{g}(x, \omega)+R e \sum_{n=1}^{N_{f}} \bar{g}\left(x, \omega+\frac{i n \pi}{t}\right)(-1)^{n}\right),
$$




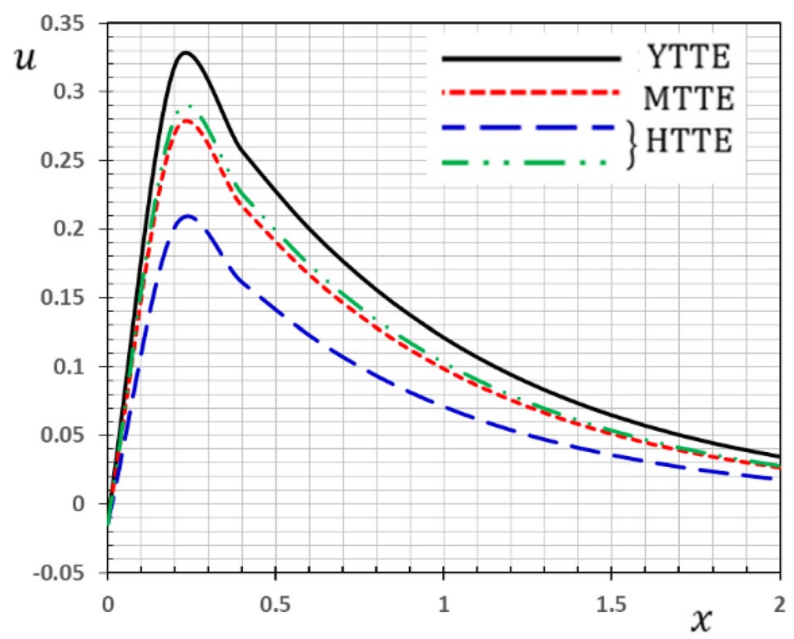

Figure 1. The displacement $u$ for different models of two temperatures.

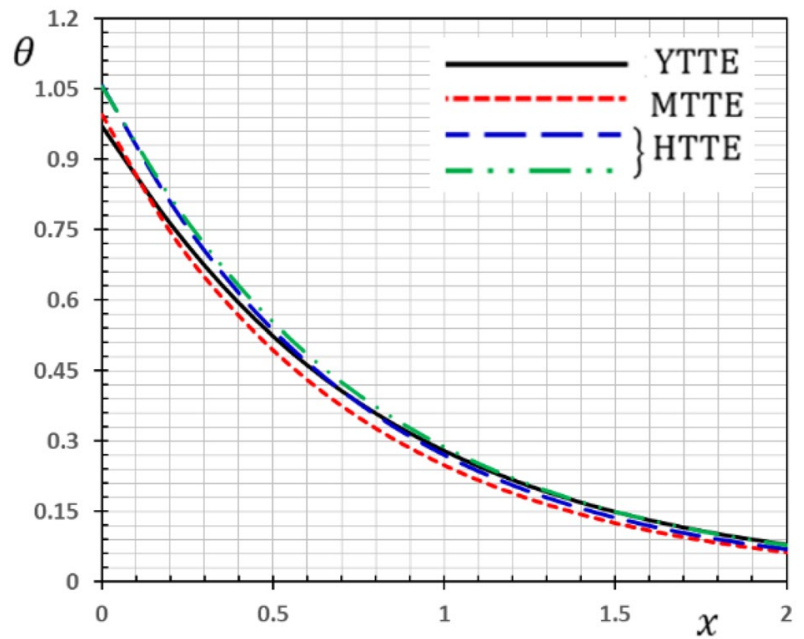

Figure 2. The thermodynamic temperature $\theta$ for different models of two temperatures.

where $N_{f}$ is a finite number of terms, $R e$ is the real part and $i$ is imaginary number unit. For faster convergence, numerous numerical experiments have shown that the value of $\omega$ satisfies the relation $\omega t \cong 4.7^{41}$. The numerical analysis were performed using the procedure proposed by ${ }^{40}$ with the help of MATHEMATICA programming.

Now we will analyze the effect of higher expansion orders $m, n, p$ and the temperature discrepancy $a$ on the physical variables. To study the influence of the higher-order time-derivatives (HOTD) $m, n, p$ as well as the distinction parameter of two-temperature $a$ on the different fields, we introduce the current numerical results in the form of tables and graphs. Note that if $a=0$ indicates the one-temperature model with HOTD and when $a=0.02 \neq 0$ indicates the two-temperature model with a higher order. The distributions of thermodynamic and conductive temperatures $\theta$ and $\varphi$, displacement $u$ and axial stress $\sigma$ are all illustrated in Figs. 1, 2, 3 and 4 and in Tables 1,2, 3 and 4 for different values of the space $x$. In this section, we compare also the numerical calculations due to the HTTE thermoelastic model to other thermoelasticity models (DPL, LS, YTTE and MTTE).

The phenomenon of limited wave propagation speeds is observed from tables and figures. Also, it is evident from tables and figures that all models display distinctly different values near the surface boundaries, and the differences decrease with increasing distance, due to the effect of thermal shock applied to the stress-free boundary. The conductive temperature profile takes the maximum value at the surface $x=0$ (thermal shock) and then gradually ultimately decreases to zero. It is detected that thermal stress $\sigma$ disappears on the surface $x=0$, which meets the state of the mechanical condition of the problem given in the Eq. (47). In Tables 1, 2, 3 and 4, various terms have been considered of the modified heat Eq. (24) and also in the equation related to the conductive and thermodynamic temperatures (25).

It can be clearly observed from the tables that the HTTE thermoelastic model gives perfect results for all HOTD parameters $(m, n, p)$. As appeared on Tables $1,2,3$ and 4 , it is enough to put $m=4, n=2, p=1$ for very 


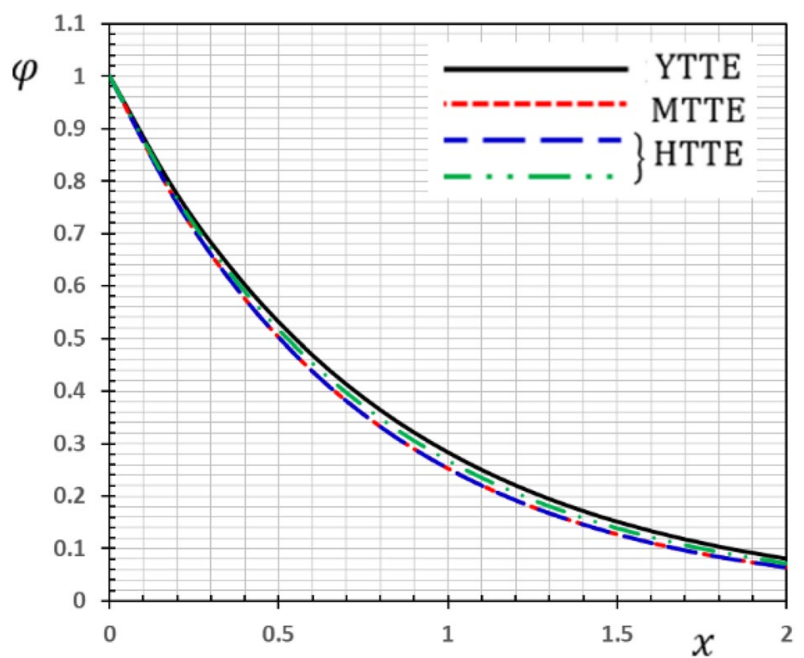

Figure 3. The conductive temperature $\varphi$ for different models of two temperatures.

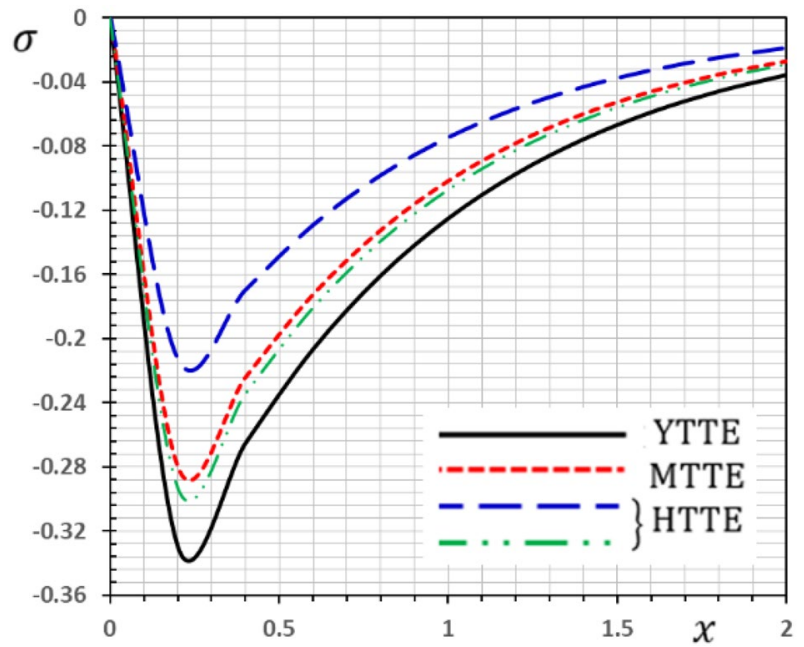

Figure 4. The stress $\sigma$ for different models of two temperatures.

\begin{tabular}{|l|l|l|l|l|l|l|l|l|l|}
\hline & & & & & \multicolumn{5}{l|}{ HTTE } \\
\cline { 8 - 10 } & LS & DPL & YTTE & MTTE & $\boldsymbol{m = 1}$ & $\boldsymbol{m}=\mathbf{2}$ & $\boldsymbol{m}=\mathbf{3}$ & $\boldsymbol{m}=\mathbf{4}$ & $\boldsymbol{m}=\mathbf{5}$ \\
\hline 0.0 & -0.01383 & -0.01383 & -0.01342 & -0.01349 & -0.01462 & -0.01458 & -0.01458 & -0.01458 & -0.01458 \\
\hline 0.2 & 0.33773 & 0.224234 & 0.318957 & 0.201787 & 0.201735 & 0.279931 & 0.281169 & 0.281293 & 0.281303 \\
\hline 0.4 & 0.272633 & 0.179314 & 0.257051 & 0.161196 & 0.161186 & 0.224568 & 0.225578 & 0.225679 & 0.225688 \\
\hline 0.6 & 0.213114 & 0.137053 & 0.200226 & 0.122816 & 0.122809 & 0.17307 & 0.173882 & 0.173963 & 0.17397 \\
\hline 0.8 & 0.166416 & 0.104538 & 0.155781 & 0.093365 & 0.09336 & 0.133167 & 0.133818 & 0.133883 & 0.133889 \\
\hline 1.0 & 0.129946 & 0.07973 & 0.121197 & 0.070969 & 0.070965 & 0.102457 & 0.102979 & 0.103031 & 0.103036 \\
\hline 1.2 & 0.101469 & 0.060809 & 0.094291 & 0.053945 & 0.053942 & 0.078829 & 0.079247 & 0.079288 & 0.079292 \\
\hline 1.4 & 0.079232 & 0.046378 & 0.073358 & 0.041005 & 0.041002 & 0.06065 & 0.060984 & 0.061017 & 0.06102 \\
\hline 1.6 & 0.061868 & 0.035372 & 0.057072 & 0.031168 & 0.031166 & 0.046663 & 0.046929 & 0.046956 & 0.046958 \\
\hline 1.8 & 0.04831 & 0.026978 & 0.044402 & 0.023692 & 0.02369 & 0.035902 & 0.036114 & 0.036135 & 0.036137 \\
\hline 2.0 & 0.037723 & 0.020575 & 0.034545 & 0.018009 & 0.018007 & 0.027623 & 0.027791 & 0.027808 & 0.02781 \\
\hline
\end{tabular}

Table 1. Effect of the higher-order time-derivatives on the displacement $u$. 


\begin{tabular}{|l|l|l|l|l|l|l|l|l|l|}
\hline & & & & \multicolumn{7}{l|}{ HTTE } \\
\cline { 8 - 10 } $\boldsymbol{x}$ & LS & DPL & YTTE & MTTE & $\boldsymbol{m}=\mathbf{1}$ & $\boldsymbol{m}=\mathbf{2}$ & $\boldsymbol{m}=\mathbf{3}$ & $\boldsymbol{m}=\mathbf{4}$ & $\boldsymbol{m}=\mathbf{5}$ \\
\hline 0.0 & 1.00028 & 1.00028 & 0.970667 & 0.97551 & 1.05677 & 1.05423 & 1.05418 & 1.05418 & 1.05418 \\
\hline 0.2 & 0.77677 & 0.760218 & 0.762884 & 0.744943 & 0.807008 & 0.817053 & 0.817222 & 0.817238 & 0.81724 \\
\hline 0.4 & 0.606432 & 0.579712 & 0.593729 & 0.566372 & 0.613558 & 0.628817 & 0.629074 & 0.6291 & 0.629102 \\
\hline 0.6 & 0.47353 & 0.442134 & 0.461924 & 0.430514 & 0.466381 & 0.48381 & 0.484105 & 0.484134 & 0.484137 \\
\hline 0.8 & 0.369756 & 0.337209 & 0.359375 & 0.327242 & 0.354505 & 0.372237 & 0.372539 & 0.372569 & 0.372572 \\
\hline 1.0 & 0.288724 & 0.257184 & 0.279592 & 0.248743 & 0.269465 & 0.286394 & 0.286684 & 0.286713 & 0.286716 \\
\hline 1.2 & 0.225451 & 0.19615 & 0.217522 & 0.189074 & 0.204825 & 0.220348 & 0.220615 & 0.220642 & 0.220644 \\
\hline 1.4 & 0.176043 & 0.149601 & 0.169231 & 0.143719 & 0.155691 & 0.169533 & 0.169773 & 0.169797 & 0.169799 \\
\hline 1.6 & 0.137464 & 0.114098 & 0.131661 & 0.109243 & 0.118343 & 0.130436 & 0.130647 & 0.130668 & 0.13067 \\
\hline 1.8 & 0.107339 & 0.087021 & 0.102432 & 0.083038 & 0.089955 & 0.100356 & 0.100538 & 0.100557 & 0.100558 \\
\hline 2.0 & 0.083815 & 0.066369 & 0.079691 & 0.063119 & 0.068376 & 0.077213 & 0.077369 & 0.077384 & 0.077385 \\
\hline
\end{tabular}

Table 2. Effect of the higher-order time-derivatives on thermodynamic temperature $\theta$.

\begin{tabular}{|l|l|l|l|l|l|l|l|l|l|}
\hline & & & & & \multicolumn{7}{l}{ HTTE } \\
\cline { 7 - 11 } & LS & DPL & YTTE & MTTE & $\boldsymbol{m}=\mathbf{1}$ & $\boldsymbol{m}=\mathbf{2}$ & $\boldsymbol{m}=\mathbf{3}$ & $\boldsymbol{m}=\mathbf{4}$ & $\boldsymbol{m}=\mathbf{5}$ \\
\hline 0.0 & 1.00028 & 1.00028 & 1.00028 & 1.00028 & 1.00028 & 1.00028 & 1.00028 & 1.00028 & 1.00028 \\
\hline 0.2 & 0.77677 & 0.760218 & 0.774147 & 0.757909 & 0.757899 & 0.766281 & 0.766422 & 0.766436 & 0.766437 \\
\hline 0.4 & 0.606432 & 0.579712 & 0.602173 & 0.576012 & 0.576002 & 0.589462 & 0.589688 & 0.589711 & 0.589713 \\
\hline 0.6 & 0.47353 & 0.442134 & 0.468485 & 0.437834 & 0.437826 & 0.453521 & 0.453786 & 0.453813 & 0.453815 \\
\hline 0.8 & 0.369756 & 0.337209 & 0.364479 & 0.332806 & 0.332799 & 0.348933 & 0.349207 & 0.349235 & 0.349237 \\
\hline 1.0 & 0.288724 & 0.257184 & 0.283563 & 0.252972 & 0.252966 & 0.268464 & 0.26873 & 0.268756 & 0.268758 \\
\hline 1.2 & 0.225451 & 0.19615 & 0.220611 & 0.192289 & 0.192284 & 0.206553 & 0.206799 & 0.206823 & 0.206825 \\
\hline 1.4 & 0.176043 & 0.149601 & 0.171634 & 0.146162 & 0.146158 & 0.158919 & 0.15914 & 0.159162 & 0.159164 \\
\hline 1.6 & 0.137464 & 0.114098 & 0.133531 & 0.111101 & 0.111097 & 0.12227 & 0.122465 & 0.122484 & 0.122486 \\
\hline 1.8 & 0.107339 & 0.087021 & 0.103886 & 0.08445 & 0.084447 & 0.094073 & 0.094242 & 0.094259 & 0.09426 \\
\hline 2.0 & 0.083815 & 0.066369 & 0.080823 & 0.064192 & 0.06419 & 0.072379 & 0.072523 & 0.072537 & 0.072539 \\
\hline
\end{tabular}

Table 3. Effect of the higher order Taylor expansions on the conductive temperature $\varphi$,

\begin{tabular}{|c|c|c|c|c|c|c|c|c|c|}
\hline \multirow[b]{2}{*}{$x$} & \multirow[b]{2}{*}{ LS } & \multirow[b]{2}{*}{ DPL } & \multirow[b]{2}{*}{ YTTE } & \multirow[b]{2}{*}{ MTTE } & \multicolumn{5}{|l|}{ HTTE } \\
\hline & & & & & $m=1$ & $m=2$ & $m=3$ & $m=4$ & $m=5$ \\
\hline 0.0 & 0 & 0 & 0 & 0 & 0 & 0 & 0 & 0 & 0 \\
\hline 0.2 & -0.34847 & -0.23475 & -0.32951 & -0.21209 & -0.2129 & -0.29123 & -0.29247 & -0.2926 & -0.29261 \\
\hline 0.4 & -0.28102 & -0.18733 & -0.26526 & -0.16903 & -0.16967 & -0.23326 & -0.23428 & -0.23438 & -0.23439 \\
\hline 0.6 & -0.21966 & -0.14317 & -0.20661 & -0.12877 & -0.12926 & -0.17976 & -0.18058 & -0.18066 & -0.18067 \\
\hline 0.8 & -0.17153 & -0.1092 & -0.16075 & -0.09789 & -0.09826 & -0.13832 & -0.13897 & -0.13904 & -0.13904 \\
\hline 1.0 & -0.13394 & -0.08329 & -0.12506 & -0.07441 & -0.07469 & -0.10642 & -0.10694 & -0.107 & -0.107 \\
\hline 1.2 & -0.10459 & -0.06352 & -0.0973 & -0.05656 & -0.05677 & -0.08188 & -0.0823 & -0.08234 & -0.08234 \\
\hline 1.4 & -0.08167 & -0.04845 & -0.0757 & -0.04299 & -0.04316 & -0.06299 & -0.06333 & -0.06337 & -0.06337 \\
\hline 1.6 & -0.06377 & -0.03695 & -0.05889 & -0.03268 & -0.0328 & -0.04847 & -0.04874 & -0.04876 & -0.04877 \\
\hline 1.8 & -0.04979 & -0.02818 & -0.04582 & -0.02484 & -0.02493 & -0.03729 & -0.0375 & -0.03753 & -0.03753 \\
\hline 2.0 & -0.03888 & -0.02149 & -0.03565 & -0.01888 & -0.01895 & -0.02869 & -0.02886 & -0.02888 & -0.02888 \\
\hline
\end{tabular}

Table 4. Effect of the higher order Taylor expansions on the stress $\sigma$.

accurate and close numerical results. Besides the first and second-order approximation, higher-order approximations, leading to higher-order DPL models, were also considered in the literature ${ }^{42}$.

Wang et al. ${ }^{43}$ investigated a well-posedness problem, given some suitable restrictions on the parameters of phase lags $\tau_{q}, \tau_{\theta}$ and $\tau_{\varphi}$. Quintanilla and Racke ${ }^{44}$ observed that there an area of influence result when $m=n$, however when $m=n+1$ they have founded some spatial approximations explaining the Phragmén-Lindelöf alternative. It is worth noting that our choices of the HOTD parameters $(m, n, p)$ are capable to involve several models of heat conduction: when we take $m=n$, we have a diffusive behavior; but by taking $m=n+1$, we get a wavelike behavior ${ }^{6,30}$. 


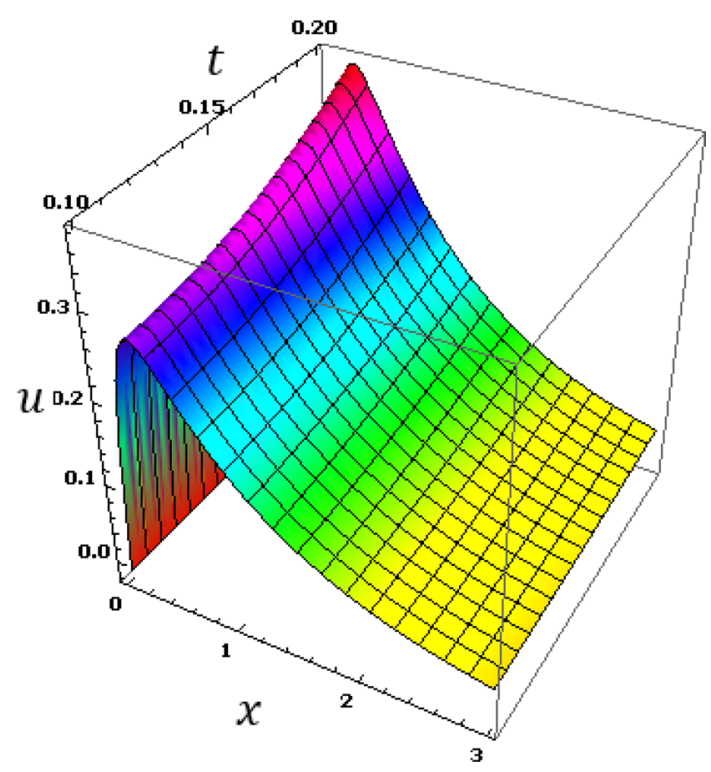

Figure 5. The displacement $u$ with different time instant $t$ and distance $x$.

Also, we have found that the HOTD parameters $(m, n, p)$ and the two-temperature parameter $a$ have a distinguished influence on the studied fields. Consequently, according to the results, it is significant to separate between the thermodynamic and the conductive temperatures. The presence of the high-order parameters increases the magnitude of the strain. The HTTE and MTTE models are largely closed to one another while the YTTE and LS models are closed to each other.

Table 1 discusses the effect of the HOTD parameters $(m, n, p)$ on the displacement $u$ for different models of thermoelasticity. It is clear from the table that the HTTE model with $(m=n=p=1)$ gives the smallest values of the displacement, while the LS theory gives the largest displacement values when we take the parameters $\tau_{q}=\tau_{0}>0, a=0, \tau_{\theta}, \tau_{\varphi} \rightarrow 0, \theta=\varphi$ and $m=1$.

The variations of the displacement $u$ versus distance $x$ for the YTTE, MTTE and HTTE models are illustrated in Fig. 1. It can be seen that the displacement beginning at $x=0$ with the minimum values for all the models and increases with $x$ to achieve the maximum value at $x=0.4$. After that, the values of the displacement $u$ drop quickly with distance.

The effect of the HOTD parameters $(m, n, p)$ on the distributions of thermodynamic temperature $\theta$ and conductive temperature $\varphi$ of the medium are displayed in Tables 2 and 3 as well as Figs. 2 and 3 for different thermoelasticity theories when the two-temperature parameter $a$ is present or absent.

From the Tables and figures, we can see that the influences of the HOTD parameters on the fields $\theta$ and $\varphi$ are very notable. Also, the parameter $a$ has a fundamental role in varying the values of the studied fields. It is noted that from Tables and Figs. 2 and 3, the variations of the fields $\theta$ and $\varphi$ is qualitatively similar for all different thermoelastic models. Moreover, it is observed that the conductive and thermodynamic temperatures increase when the high-order approximations parameters decrease. Also, the HTTE model displays the temperature field values compared to the LS, DPL, YTTE and MTTE models.

In the last set of 3D graphs (5-8), the numerical results of studied variables are introduced along the axial axis $(0.0 \leq x \leq 3.0)$ and for different time $(0.0 \leq t \leq 0.2)$ under the HTTE model. The HTTE theory with $m=4, n=3, p=2$ is used in all the $3 \mathrm{D}$ figures. Figure 5 displays the variation of the displacement profile $n$ against the axial distance due to the presence of time effects. The displacement profile grows to reach its maximum value and then decomposes as we move away from the boundary.

Observing the various results, we find that due to the presence of time, it was observed that the profiles of displacement, conductive and thermodynamic temperatures fields increase with time, which supports the physical reality. A general observation from all these numerical results and graphs (5-8), it is noted that all physical fields are sensitive to the time that is included. Figure 8 shows that the thermal stress field has an equal starting point with a value of zero, which indicates that the mechanical boundary condition is satisfied. Figure 6 displays the conductive temperature variance with distance $x$ and time $t$. From Fig. 7, we can see that all the profiles of $\varphi$ have a coincident beginning point with value $\varphi=1$, which satisfies the thermal boundary conditions (Fig. 8).

\section{Conclusions}

In the current paper, a modified two-temperature thermoelasticity model with higher-order-time derivatives (HOTD) and three different phase-lags has been constructed. The derived model was established by taking into account the Taylor series expansions of Fourier's heat conduction and the relation for the two temperatures and keeping terms up to appropriate higher-orders in the phase-lags $\tau_{q}, \tau_{\varphi}$ and $\tau_{\theta}$. Also, the two temperature model with one relaxation time (YTTE) and two temperature theory with two phase-lags (MTTE) are compared with the higher-order model with two-temperature and three-phase-lags. 


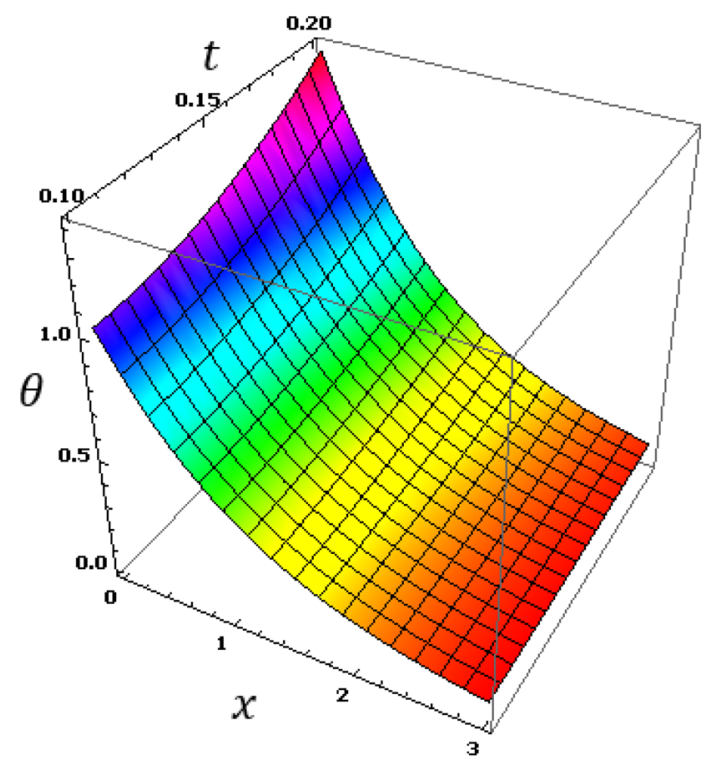

Figure 6. The thermodynamic temperature $\theta$ with different time $t$ and distance $x$.

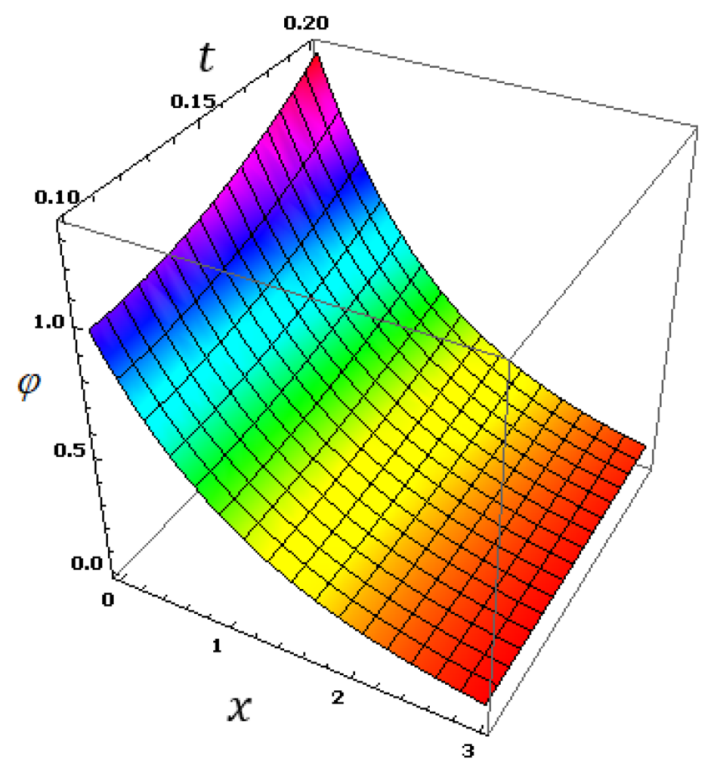

Figure 7. The conductive temperature $\varphi$ with different time instance $t$ and distance $x$.

To validate the proposed model and show that it is more accurate, the results are tabulated. From the Tables and figures, we observed that the effects of the HOTD parameters on $\theta$ and $\varphi$ fields are very significant. The sensitivity of the physical fields to the variation of the high-order parameters is investigated. For the current HTTE model, $m=4$ is sufficient to obtain valid and effective results.

It is also evident from the results that when the HOTD parameters are lower than or equal to four, the relevant model can be thermodynamically compatible, providing that it makes suitable appropriate assumptions upon the delay times.

This result is consistent with the results got by Chiriţă ${ }^{25,31}$. Finally, this work describes that the theory of thermoelasticity with two-temperature and three-phase-lags and HOTD parameters explains the behavior of the particles of the thermoelastic body more realistically than the model of thermoelasticity with two-temperature with one or two phase-lags. Also, the current study helps some researchers to show how they choose the values of these parameters. 


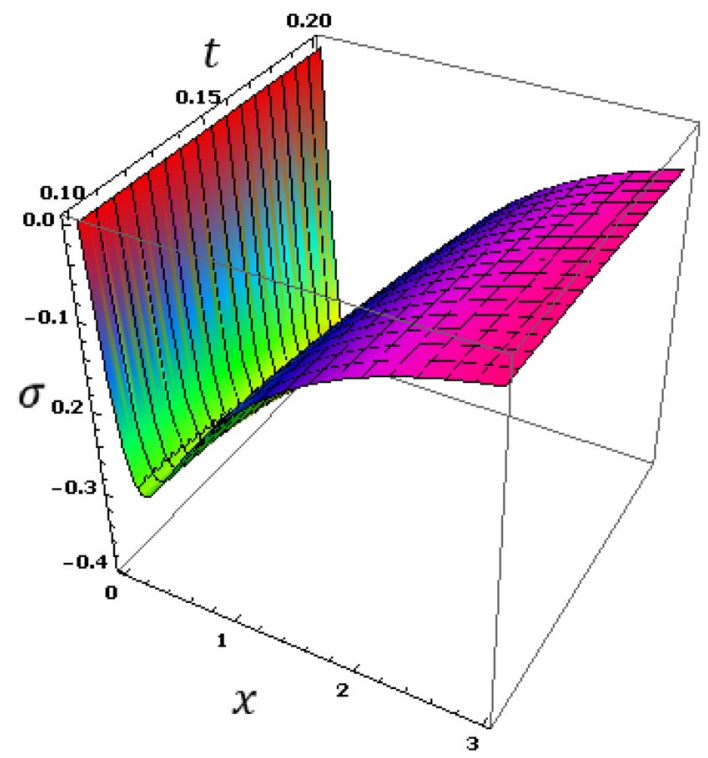

Figure 8. The stress $\sigma$ with different time instance $t$ and distance $x$.

Received: 15 March 2020; Accepted: 23 July 2020

Published online: 12 August 2020

\section{References}

1. Biot, M. Thermoelasticity and irreversible thermodynamics. J. Appl. Phys. 27, 240-253 (1956).

2. Green, A. E. \& Lindsay, K. A. Thermoelasticity. J. Elast. 2(1), 1-7 (1972).

3. Tzou, D. Y. A unified filed approach for heat conduction from macro to macroscales. ASME J. Heat Transf. 117, 8-16 (1995).

4. Chandrasekharaiah, D. S. Hyperbolic thermoelasticity: a review of recent literature. Appl. Mech. Rev. 51, 705-729 (1998).

5. Tzou, D. Y. The generalized lagging response in small-scale and high-rate heating. Int. J. Heat Mass Transf. 38, 3231-3234 (1995).

6. Tzou, D. Y. Experimental support for the lagging behavior in heat propagation. J. Thermophys. Heat Transf. 9, 686-693 (1995).

7. Chen, P. J. \& Gurtin, M. E. On a theory of heat conduction involving two temperatures. Z. Angew. Math. Phys. 19(4), 614-627 (1968).

8. Gurtin, M. E. \& Williams, W. O. On the Clausius-Duhem inequality. Z. Angew. Math. Phys. 17(5), 626-633 (1966).

9. Gurtin, M. E. \& Williams, W. O. An axiomatic foundation for continuum thermodynamics. Arch. Ration. Mech. Anal. 26(2), 83-117 (1967).

10. Chen, P. J., Gurtin, M. E. \& Williams, W. O. A note on nonsimple heat conduction. Z. Angew. Math. Phys. 19(6), 969-970 (1968).

11. Chen, P. J., Gurtin, M. E. \& Williams, W. O. On the thermodynamics of non-simple elastic materials with two temperatures. $Z$. Angew. Math. Phys. 20(1), 107-112 (1969).

12. Warren, W. E. \& Chen, P. J. Wave propagation in the two temperature theory of thermoelasticity. Acta Mech. 16(1-2), 21-33 (1973).

13. Puri, P. \& Jordan, P. M. On the propagation of harmonic plane waves under the two-temperature theory. Int. J. Eng. Sci. 44(17), 1113-1126 (2006).

14. Kumar, R. \& Mukhopadhyay, S. ffects of thermal relaxation time on plane wave propagation under two-temperature thermoelasticity. Int. J. Eng. Sci. 48, 128-139 (2010).

15. Deswal, S. \& Kalkal, K. K. Three-dimensional half-space problem within theframework of two-temperature thermo-visco-elasticity with three-phase-lag effects. Appl. Math. Model. 39(23), 7093-7112 (2015).

16. Singh, B. Wave propagation in a rotating transversely isotropic two-temperature generalized thermo elastic medium without dissipation. Int. J. Thermophys. 37(1), 1-13 (2016).

17. Kumar, R., Vashisth, A. K. \& Ghangas, S. waves in anisotropic thermoelastic medium with phase lag, two-temperature and void. Mater. Phys. Mech. 35, 126-138 (2018).

18. Abouelregal, A. E. \& Zenkour, A. M. Two-temperature thermoelastic surface waves in micropolar thermoelastic media via dualphase-lag model. Adv. Aircr. Spacecr. Sci. 4(6), 711-727 (2017).

19. Quintanilla, R. On existence, structural stability, convergence and spatial behavior in thermoelasticity with two temperatures. Acta Mech. 168(1-2), 61-73 (2004).

20. Youssef, H. M. Theory of two-temperature-generalized thermoelasticity. IMA J. Appl. Math. 71, 383-390 (2006).

21. Mukhopadhyay, S., Prasad, R. \& Kumar, R. On the theory of two-temperature thermoelasticity with two phase-lags. J. Therm. Stress. 34(4), 352-365 (2011).

22. Miranville, A. \& Quintanilla, R. On the spatial behavior in two-temperature generalized thermoelastic theories. Z. Angew. Math. Phys. 68, 110-125 (2017).

23. Kumar, R., Miglani, A. \& Rani, R. Generalized two temperatures thermoelasticity of micropolar porous circular plate with three phase lag model. J. Mech. 34(6), 779-789 (2018).

24. Abbas, I. A. \& Youssef, H. M. Two-temperature generalized thermoelasticity under ramp-type heating by finite element method. Meccanica 48(2), 331-339 (2013).

25. Abbas, I. A. \& Youssef, H. M. A nonlinear generalized thermoelasticity model of temperature-dependent materials using finite element method. Int. J. Thermophys. 33(7), 1302-1313 (2012).

26. Banik, S. \& Kanoria, M. Effects of three-phase-lag on two-temperature generalized thermoelasticity for infinite medium with spherical cavity. Appl. Math. Mech. Engl. Ed. 33(4), 483-498 (2012).

27. Abouelregal, A. E. Modified fractional thermoelasticity model with multi-relaxation times of higher order: application to spherical cavity exposed to a harmonic varying heat. Waves Random Complex Media https://doi.org/10.1080/17455030.2019.1628320 (2019). 
28. Abouelregal, A. E. Two-temperature thermoelastic model without energy dissipation including higher order time-derivatives and two phase-lags. Mater. Res. Express 6, 116535 (2019).

29. Abouelregal, A. E. On Green and Naghdi thermoelasticity model without energy dissipation with higher order time differential and phase-lags. J. Appl. Comput. https://doi.org/10.22055/JACM.2019.29960.1649 (2019).

30. Chiriţă, S. On high-order approximations for describing the lagging behavior of heat conduction. Math. Mech. Solids 24(6), 108128651875835 (2019).

31. Baron Fourier, J. B. J. The analytical theory of heat (The University Press, London, 1878).

32. Quintanilla, R. A well posed problem for the dual-phase-lag heat conduction. J. Therm. Stress. 31, 260-269 (2008).

33. Quintanilla, R. Exponential stability in the dual-phase-lag heat conduction theory. J. Non-Equilib. Thermodyn. 27, 217-227 (2002).

34. Fabrizio, M. \& Franchi, F. Delayed thermal models: stability and thermodynamics. J. Therm. Stress. 37, 160-173 (2014).

35. Chiriţă, S., Ciarletta, M. \& Tibullo, V. On the wave propagation in the time differential dual-phase-lag thermoelastic model. Proc. R. Soc. A 471, 20150400 (2015).

36. Chiriță, S. On the time differential dual-phase-lag thermoelastic model. Meccanica 52, 349-361 (2017).

37. Chiriţă, S., Ciarletta, M. \& Tibullo, V. On the thermomechanic consistency of the time differential dual-phase-lag models of heat conduction. Int. J. Heat Mass Transf. 114, 277-285 (2017).

38. Lord, H. W. \& Shulman, Y. H. A generalized dynamical theory of thermoelasticity. J. Mech. Phys. Solids. 15(5), 299-309 (1967).

39. Zenkour, A. M. \& Abouelregal, A. E. The fractional effects of a two-temperature generalized thermoelastic semi-infinite solid induced by pulsed laser heating. Arch. Mech. 67(1), 53-73 (2015).

40. Honig, G. \& Hirdes, U. A method for the numerical inversion of the Laplace transform. J. Comput. Appl. Math. 10, 113-132 (1984).

41. Tzou, D. Y. Macro- to Micro-scale Heat Transfer: The Lagging Behavior, Vol. 471, No. 2183, 20150400 (Wiley, Chichester, 2015).

42. Askarizadeh, H., Baniasadi, E. \& Ahmadikia, H. Equilibrium and non-equilibrium thermodynamic analysis of high-order dualphase-lag heat conduction. Int. J. Heat Mass Transf. 104, 301-310 (2017).

43. Wang, L. \& Xu, M. Well-posedness of dual-phase-lagging heat equation: higher dimensions. Int. J. Heat Mass Transf. 45, 1165-1171 (2002).

44. Quintanilla, R. \& Racke, R. Spatial behavior in phase-lag heat conduction. Differ. Integr. Equ. 28, 291-308 (2015).

\section{Author contributions}

All author in this research have completely prepared it and there are no other authors involved in preparing this research.

\section{Competing interests}

The authors declare no competing interests.

Additional information

Correspondence and requests for materials should be addressed to A.E.A.

Reprints and permissions information is available at www.nature.com/reprints.

Publisher's note Springer Nature remains neutral with regard to jurisdictional claims in published maps and institutional affiliations.

c) (i) Open Access This article is licensed under a Creative Commons Attribution 4.0 International License, which permits use, sharing, adaptation, distribution and reproduction in any medium or format, as long as you give appropriate credit to the original author(s) and the source, provide a link to the Creative Commons license, and indicate if changes were made. The images or other third party material in this article are included in the article's Creative Commons license, unless indicated otherwise in a credit line to the material. If material is not included in the article's Creative Commons license and your intended use is not permitted by statutory regulation or exceeds the permitted use, you will need to obtain permission directly from the copyright holder. To view a copy of this license, visit http://creativecommons.org/licenses/by/4.0/.

(c) The Author(s) 2020 